\title{
Models of brain injury vocational rehabilitation: The evidence for resource facilitation from efficacy to effectiveness
}

\author{
Lance E. Trexler* and Devan R. Parrott \\ Rehabilitation Hospital of Indiana, Indiana University School of Medicine, Indianapolis, IN, USA
}

Revised/Accepted October 2017

\begin{abstract}
.
BACKGROUND: Resource Facilitation (RF) is an intervention developed to improve return to work (RTW) following brain injury. RF is an individualized treatment specializing in connecting patients and caregivers with community-based resources and services to mitigate barriers to return to work.

OBJECTIVES: Examine the effectiveness of the RHI RF program for a clinical prospective cohort of participants referred to this program from the State Vocational Rehabilitation agency.

METHODS: Participants were 243 participants with data drawn from the two sources: 33 from previous randomized controlled trial (RCT) control groups who did not receive RF and 210 from clinical patients discharged from the RHI RF program.

RESULTS: At discharge from RF, a greater proportion of the treatment group obtained employment than the control group $\left[\mathrm{X}_{(1)}^{2}=5.39, p=0.018\right]$. When controlling for baseline level of disability, treatment group significantly predicted employment outcome (Wald $=4.52, p=0.033$ ) and participants in the treatment group were 2.3 times more likely to return to work than controls.

CONCLUSIONS: Previous RCTs have studied the RHI RF model and demonstrated significant efficacy. The findings from the present study are consistent with the employment rates found in the previous RCT's following RF, and also provide initial support for the clinical effectiveness of RF.
\end{abstract}

Keywords: Brain injuries, return to work, employment, rehabilitation, vocational

\section{Return to work (RTW) after acquired brain injury (ABI)}

Return to work after brain injury has always been regarded as a critical outcome metric in research, yet established continuums of rehabilitation services typically do not extend through vocational placement

\footnotetext{
*Address for correspondence: Lance E. Trexler, PhD, FACRM, Rehabilitation Hospital of Indiana, Indiana University School of Medicine, 9531 Valparaiso Court, Indianapolis, IN 46268, USA. Tel.: +1 317329 2411; Fax: +1 317872 0914; E-mail: lance.trexler@rhin.com.
}

and follow-up. When vocational services do exist, they are often a) not brain injury specialized or b) integrated into an existing continuum of services, which typically results in people not receiving vocational rehabilitation services, or if they do, it is many years post-injury. In addition to the brain injury specific barriers to RTW, these system barriers compromise vocational outcome.

A variety of studies have demonstrated that approximately $30-40 \%$ of people with ABI ultimately return to work. A systematic review (Van Velzen, Van Bennekom, Edekaar, Sluiter, \& 
Frings-Dresen, 2009) found that $40.8 \%$ of those with traumatic brain injury (TBI) and $39.3 \%$ with non-TBI returned to work. Inclusion criteria in this review included a) the subjects were working prior to their injury, b) were aged 18-65 years, and c) return to work was an outcome measure. Their review was based on a sample of 49 studies that met all of the inclusion criteria. It should be noted however that this study did not separate outcomes based on severity of injury.

A more recent study was completed using population estimates from the Traumatic Brain Injury Model Systems National Database (TBIMS-NDB) (Cuthbert et al., 2015). In this study, the investigators culled 3121 subjects from the database which were weighted to obtain population estimates to match the US TBI rehabilitation population based on both the Uniform Data System for Medical Rehabilitation (UDS, 2012) and the American Medical Rehabilitation Providers Association, eRehab (American Medical Rehabilitation Providers Association, 2012). Their sample was based on inclusion criteria that included less than 60 at the age of injury, not retired at injury, and alive two years postinjury. These investigators found that $39.6 \%$ were employed, which included paid legal or illegal work, with or without accommodations. Of these $39.6 \%$ that were able to RTW, $65 \%$ of them were employed full time.

A number of studies have found that severity of brain injury has a significant impact on return to work. For example, in 2002, Groswasser et al. found that $84 \%$ of subjects with mild TBI were able to RTW, and in another study of mild TBI, $78 \%$ were able to RTW (Hanlon et al., 1999). Dikmen and colleagues (Dikmen, Temkin, Machamer, Holubkov, Fraser, \& Winn 1994) found that 37 percent of subjects with severe traumatic brain injury (TBI) had returned to work, 64 percent with moderate TBI, and 83 percent of mild TBI returned to work at two years post-injury.

Even these findings may however over-estimate rates of RTW for moderate and severe brain injury. Certainly not all people who need inpatient rehabilitation receive these services, and consequently, are not included into the TBIMS database. Individuals who are seen in their State Vocational Rehabilitation agency are typically many years post-injury, did not have access to specialized brain injury rehabilitation services, have developed multiple co-morbidities, have lost vocational skills and networks, in addition to presenting with persisting cognitive and neurobehavioral disabilities (often among others), making return to work a certain challenge. For example, Schopp, Johnstone, Unger, \& Goldman (2003) found that only 18 percent of State Vocational Rehabilitation clients with TBI were successfully placed.

\section{Barriers to RTW after acquired brain injury}

Vocational rehabilitation of people with brain injury represents a significant challenge from all perspectives. Barriers to RTW span across individual and family variables, as well as social, environmental, and system/organizational domains. It is for these reasons that an effective approach to RTW following brain injury will not be a "medical" or a "vocational" model, but rather one that is capable of responding to the unique and individualized constellation of barriers, and the interactions that each case presents.

Critical individual barriers include neurobehavioral impairments (e.g., disinhibition, impulsivity, decreased initiation) or cognitive impairments (e.g., impaired goal setting or task monitoring, memory, attention) that are ubiquitous following $\mathrm{ABI}$ and can significantly affect job performance and adjustment in the workplace (Dikmen et al., 1994). Medical consequences of brain injury (e.g., posttraumatic seizures) or co-morbidities (e.g., depression or substance abuse) also represent barriers to RTW or work stability. Level of behavioral adjustment post-injury affect family adjustment (Kreutzer, Marwitz, \& Kepler, 1992), which in turn influence recovery and adaptation. Further, changes in social roles typically result in increased emotional and economic burden for family members, making it even more difficult to sustain family advocacy and emotional engagement, as well as support for the family member with the brain injury (Kolakowsky-Hayner \& Kishore, 1999; Kreutzer, Gervasio, \& Camplair, 1994).

Social barriers include lack of reimbursement for services and the absence of brain injury expertise among the host of providers involved in the vocational rehabilitation of people with brain injury. Multiple providers (e.g., employment services and cognitive rehabilitation) do not typically collaborate, compromising the potential effectiveness of each of their interventions. Awareness of State agency resources is typically lacking, and access to these resources can be cumbersome and overwhelming. Further, States do not typically have an organized State plan for brain injury, resulting in service silos within and between State agencies with resulting service gaps. 
Complicating the vocational rehabilitation process even more is the fact that, at least in moderate to severe TBI, most people will have chronic disability that is variable in type and severity over time (Corrigan \& Hammond, 2013). In fact, the severity of TBI disability has been found to vary more often that it remains static in long-term follow-up studies. Further, the individual and social-environmental variables dynamically interact with each other, where, for example, a loss of a significant relationship can result in depression that, in turn, results in further impairment of memory, which then results in impaired vocational performance. On the other hand, successful cognitive rehabilitation may result in new compensatory strategies, that when integrated into the work setting, with improvement in self-efficacy, and then a better response to psychotherapy for depression. Again, brain injury vocational rehabilitation is neither medical nor vocational; it is of necessity an individualized process of eliminating or mitigating brain injury-specific and other barriers with a holistic scope.

\section{Models of vocational rehabilitation for acquired brain injury}

A systematic review of the literature on different types of vocational rehabilitation for people with ABI (Fadyl \& McPherson, 2009) demonstrated that there have been essentially three different approaches that included a supported employment (SE) model, a "Program-based vocational rehabilitation model" often referred to as a comprehensive day treatment program (CDT) model, and a "vocational case coordination (VCC)" model. The development of SE for people with brain injury was a significant contribution to improving vocational outcome (Wehman et al., 1989; Wehman, Bricout, \& Targett, 2000; Wehman et al., 2003). Wehman and colleagues modified the individual placement model for people with brain injury and there was a clear emphasis on individualized training at the work site through a job coach rather than through pre-placement training and intervention. In contrast, the CDT program provided individual and group treatment for cognitive, neurobehavioral, and psychological difficulties driven by a neuropsychological approach (Ben-Yishay, Silver, Piasetsky, \& Rattock, 1987; Prigatano et al., 1994). These programs were typically provided services four or five days a week for approximately six months and included family education and training, as well as vocational trials, placement and follow-up. The first two studies on "vocational case coordination," the third approach, were completed by Malec and colleagues (Malec, Buffington, Moessner, \& Degiorgio, 2000; Malec \& Moessner, 2006). This approach features an individualized approach to promoting access to vocationally-related needs and ensuring integration of services to impact on vocational skills and workplace adaptation. The overall structure of these different models from Fadyl and McPherson (2009) are provided in Fig. 1.

These investigators also rated the quality of the research for each of these models to determine the relative strength of experimental support for their effectiveness. Their findings in this respect are summarized in Table 1.

The research by Malec and colleagues (2000, 2005) on VCC and the systematic review by Fadyl and McPherson (2009) provided the scientific background for the development of the RF model developed at the Rehabilitation Hospital of Indiana (RHI).

\subsection{The RHI resource facilitation model}

The clinical research team at RHI incorporated the findings from a best practices guide from 16 RF programs in the United States completed by the then Brain Injury Association on "Resource Facilitation" into the VCC model (Brain Injury Association, 2001). RF was defined as "a partnership that helps individuals and communities choose, get and keep information, services and supports to make informed choices and meet their goals. The collaborative process involves participants (individuals with brain injury and their personal support systems) working in partnership with facilitators (individuals who provide assistance in navigating systems) to achieve agreed upon goals" (p. 2). This guide provided information on best practices while the research on VCC provided evidence to inform the RHI RF model. Like these 16 RF programs, RHI's development of RF program began with funding in 2009 from the Health Resources and Services Administration (HRSA).

The efficacy of the RHI RF model has been investigated through two randomized controlled trials (RCT). In the first RCT (L.E. Trexler, L.C. Trexler, Malec, Klyce, \& Parrott, 2010), 22 subjects with ABI were recruited either while in the acute rehabilitation unit or shortly thereafter. It was found that the RF group had a successful return to work rate of 64 percent compared to 36 percent in the control 


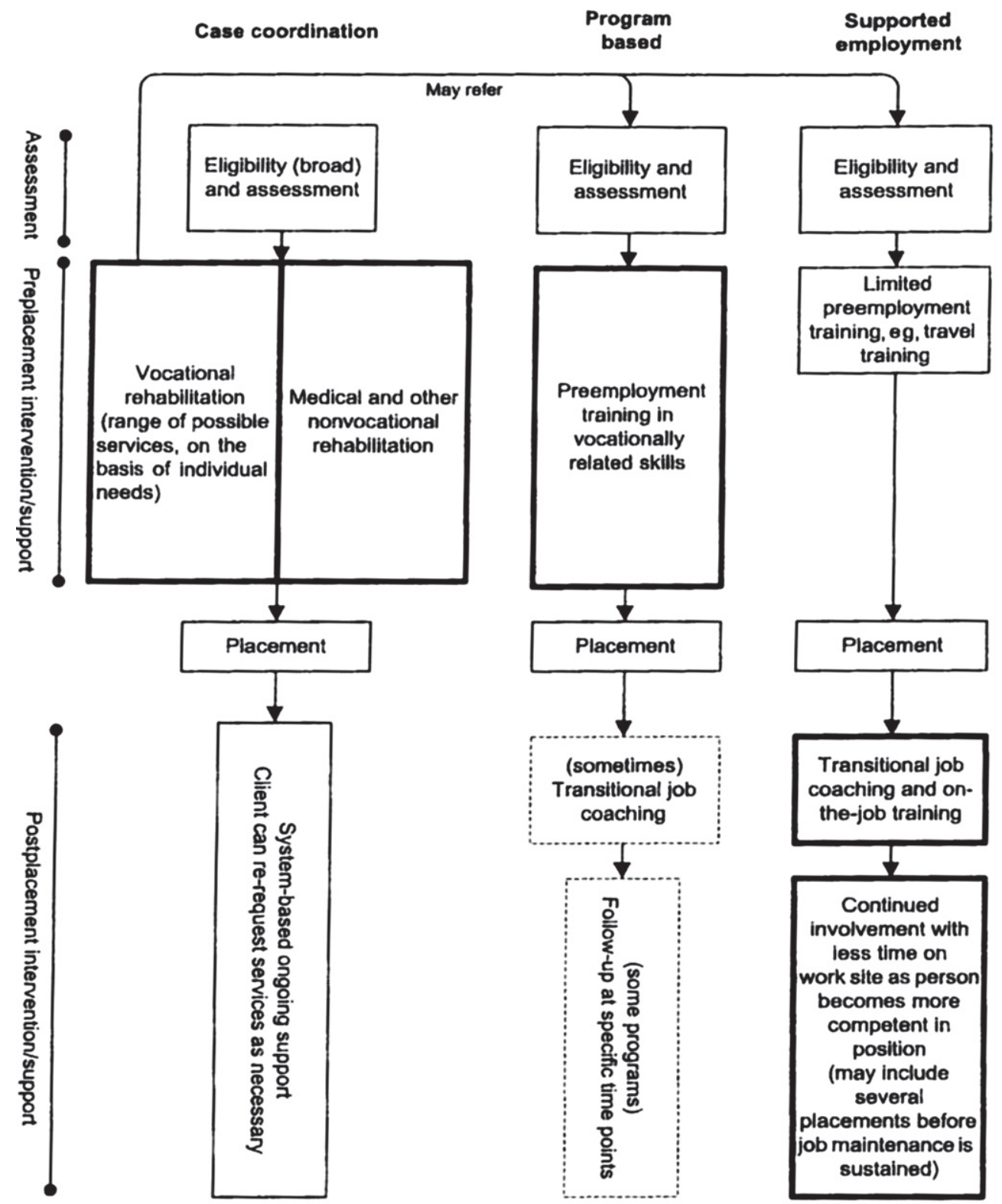

Fig. 1. Overview of the structure of three different types of vocational rehabilitation programs for brain injury (reproduced from Fadyl and McPherson, 2009 with permission).

group (Wald-Wolfkowitz $z=-3.27, P<0.0001$ ). Further, the RF group was found to improve significantly more than the control on a measure of participation in activities at home and in the community relative to controls $(F=9.11, P<0.007)$. A larger RCT of 44 subjects with $\mathrm{ABI}$, again recruited while in an acute rehabilitation unit or shorter after being discharged, demonstrated that 69 percent of the RF group returned to employment compared 50 percent in the control group (Trexler, Parrott, \& Malec, 2015).
In addition, logistic regression analyses revealed that treatment group was a significant predictor of outcome (Wald $=4.91, P=0.027$ ), and RF participants with a goal of returning to work had 7 times higher odds of returning to productive activities relative to controls (95\% confidence interval, $(1.25,39.15)$ ). Based on these findings and the support for the efficacy of the RHI RF model, the Indiana Vocational Rehabilitation Services supported a prospective clinical cohort study to examine the effectiveness of 
Table 1

Conclusions regarding the strength of the evidence for different types of vocational rehabilitation programs for brain injury

\begin{tabular}{llc}
\hline $\begin{array}{l}\text { Comprehensive Day } \\
\text { Treatment (CDT) }\end{array}$ & Supported Employment (SE) & $\begin{array}{c}\text { Vocational Case Coordination } \\
\text { (VCC) }\end{array}$ \\
\hline $\begin{array}{l}\text { Weak evidence for better } \\
\text { vocational outcomes for } \\
\text { those with TBI with CDT }\end{array}$ & $\begin{array}{c}\text { Weak evidence that SE } \\
\text { "allows some individuals } \\
\text { program }\end{array}$ & $\begin{array}{c}\text { Moderate evidence that VCC } \\
\text { "produces higher } \\
\text { employment and } \\
\text { productivity outcomes" }\end{array}$ \\
$\begin{array}{l}\text { employed postinjury to be } \\
\text { employed specifically } \\
\text { within the supported } \\
\text { employment model" }\end{array}$ & $\begin{array}{c} \\
\text { Weak evidence that } \\
\text { participants who receive SE } \\
\text { employment is maintained } \\
\text { approximately 50\%) }\end{array}$ & $\begin{array}{c}\text { Weak evidence that those who } \\
\text { received VCC earlier after } \\
\text { injury are employed earlier }\end{array}$ \\
& days & \\
\hline
\end{tabular}

RF. Further, as this trial was supported by the State Vocational Rehabilitation agency through referral of clients for RF, goals could be return to post-secondary education, although this was an infrequent goal.

\section{Objectives}

The overall objective of this study was to examine the effectiveness of the RHI RF program for a clinical prospective cohort of participants referred to this program from the Indiana Vocational Rehabilitation Services. Based on our previous research and previous research, we had two hypotheses that included 1) the participants in the RF prospective clinical cohort would demonstrate a significantly better rate of RTW or school, and 2) group assignment (RF versus controls) would predict outcome with baseline level of disability as a covariate.

\section{Methods}

\subsection{Study design and population}

The present study examined the vocational outcome for 243 participants (163 men and 80 women; mean age $38.59 \pm 13.05$ years) with data drawn from the two sources: 33 from the RCT control groups who did not receive RF and 210 from clinical patients discharged from the RHI RF program. Since the samples were from two different sources, the inclusion/exclusion criteria are different for each sample. For the control group, the inclusion criteria for the RCT was: a) TBI or diffuse encephalopathy including metabolic, infectious or toxic (but not due to alcohol abuse) encephalopathy, or intracranial hemorrhage, b) between 18-60 years old, c) English as a native language or non-native speaker with the assistance of a relative who is an English speaker or a translator, d) the individual with a brain injury had been employed at the time of injury, e) the individual had a returnto-work goal after the injury, and (f) participant or legal proxy consents to study participation. Exclusion criteria included: a) the presence of acute psychosis or the emergence of psychosis during the course of the study and b) history of treatment received for substance abuse within the preceding two years.

The obvious inclusion criteria for this clinical sample included a) a diagnosis of an acquired brain injury and b) a return to work or post-secondary school goal, or they would have not been appropriate clients of the State Vocational Rehabilitation agency. No explicit inclusion/exclusion criteria were applied to the clinical cohort sample. In general, participants who may have had an active psychosis were very likely excluded and some participants with alcohol or drug abuse to an extent to which would interfere with goal attainment were not admitted into RF. We also did not recommend RF for a few clients that had very severe physical, cognitive, and neurobehavioral impairment to an extent that the relative probability that they could become competitively and independently employed was very remote.

The duration of RF was on average nine months prior to the participant becoming competitively employed. Participants were then followed for 90 days to ensure that vocational supports were sustainable and that their employment adjustment was stable.

\subsection{Measures}

Return to either part- or full-time competitive work or post-secondary school was the main outcome 
measure, and data for the number of hours worked per week and type of work was available for $66 \%$ of the treatment sample that was successful for return to work or school. A successful outcome was recorded when the participant was able to sustain employment or return to school for the entirety of the interval between place and follow-up 90 days thereafter.

The Mayo-Portland Adaptability Inventory - 4 (MPAI-4) was designed to measure post-acute disability level in persons with brain injury (Malec, 2005). Change is MPAI-4 is also used to evaluate the effectiveness of rehabilitation programs. It consists of 28 total items and subscales measure cognitive and physical abilities, psychosocial adjustment and participation in activities at home and in the community. This measure was used as a covariate to determine if group assignment would predict employment outcome.

\subsection{Statistical analyses}

The present retrospective case-control study used control data from our previous RCT's as well as group data from a current clinical cohort who received RF services. Propensity scores were not utilized as the control group data were from subjects previously randomized to a control condition. However, baseline comparisons were used to ensure the groups were not statistically different from each other prior to treatment.

All analyses were completed using IBM SPSS version 24. Independent sample $t$-tests and Chi-square analyses were used to compare baseline variables between the two groups. A Chi-square analysis was used to compare the success rate in each group and logistic regression was used to predict employment status based on group assignment. Nagelkerke $\mathrm{R}^{2}$ was used to estimate explained variance. There were no outliers, as assessed by examination of studentized residuals for values greater than three standard deviations. Effect size is reported as partial $\eta^{2}$. An effect size less than 0.05 was considered small; moderate, when between 0.05 and 0.25 ; moderately large, when between 0.25 and 0.50 ; and large when greater than 0.50 . Significance levels were set at $p<0.05$ and Bonferroni corrections were used to correct for multiple pairwise comparisons.

\section{Results}

Some very noteworthy demographic differences between the two groups were present at baseline: years post injury, age at injury, and injury type (see Table 2). As previously noted, the control group was derived from a previous RCT where subjects were recruited while in acute inpatient rehabilitation or shortly thereafter, and were a little more than two months post-injury. In marked contrast, the participants in the RF group in the present study were referred from Indiana Vocational Rehabilitation Services and were found to be, on average, over 9 years post-injury. Additionally, the age at injury for the RF group was found to be significantly younger (29.6 years) as compared to the control group which was on average 40 years old. This difference is likely attributable to significant differences between the two groups in terms of diagnosis, where there were more participants with stroke in the control group and more moderate to severe TBI subjects in the RF group.

\subsection{Hypothesis 1: Success rate by group}

Of the 210 participants in the treatment group, $69 \%$ $(n=145)$ were successful for return to competitive work. Six of these 145 successful outcomes were for participants that had a goal of return to school. Of the portion of the sample for which work hours and type of employment information was available, it was found that the average hours worked per week was $24.88(\mathrm{sd}=10.38)$ and $36 \%$ of the successfully closed cases were full-time (30+ hours per week). The occupations to which they were placed are provided in Fig. 2. Almost half of the successful participants returned to either administrative support or laborer positions, but the other half were distributed across all other types of occupations. Of the 33 participants in the control group, $48 \%(n=16)$ successfully returned to paid employment. This difference was significant, indicating a greater proportion of success in the treatment group than the control at the end of the treatment duration $\left[\mathrm{X}^{2}{ }_{(1)}=5.39, p=0.018\right]$ (see Fig. 3).

\subsection{Hypothesis 2: Treatment group predicts outcome with baseline level of disability as a covariate}

The first model examined the relationship between baseline level of disability and employment. A logistic regression model was statistically significant, $\chi^{2}(1)=9.92, p=0.002$, but it should be noted that this model only explained $5.5 \%$ of the variance in productive activity and correctly classified $67.9 \%$ of the cases. Level of disability at baseline provided an exponential slope of 0.94 indicating that with every 
Table 2

Patient characteristics and outcomes by group

\begin{tabular}{lccr}
\hline Characteristic & Resource Facilitation $(n=210)$ & Controls $(n=33)$ & $p$ \\
\hline & Mean \pm standard deviation & Mean \pm standard deviation & \\
Age (y) & $38.32(13.28)$ & $40.30(11.54)$ & 0.419 \\
Years Post Injury & $9.61(10.30)$ & $0.18(0.10)$ & 0.000 \\
Age at Injury & $29.61(15.58)$ & $40.30(11.54)$ & 0.000 \\
Years of education & $13.24(2.21)$ & $13.46(2.24)$ & 0.610 \\
Baseline MPAI & $42.36(7.97)$ & $43.70(8.25)$ & 0.373 \\
& Frequency $(\%)$ & Frequency $(\%)$ & \\
Sex & $142(68 \%)$ & $21(64 \%)$ & \\
Male & $68(32 \%)$ & $12(36 \%)$ & 0.692 \\
Female & & & \\
Race & $191(91 \%)$ & $33(100 \%)$ & \\
White & $16(7.6 \%)$ & & \\
African American & $3(1.4 \%)$ & & \\
Hispanic & $2(1 \%)$ & -085 \\
Diagnosis & $152(72 \%)$ & $17(52 \%)$ & \\
Mild TBI & $33(16 \%)$ & $1(3 \%)$ & \\
Moderate to Severe TBI & $23(11 \%)$ & & 0.001 \\
Stroke & & & \\
Other & & & \\
\hline
\end{tabular}

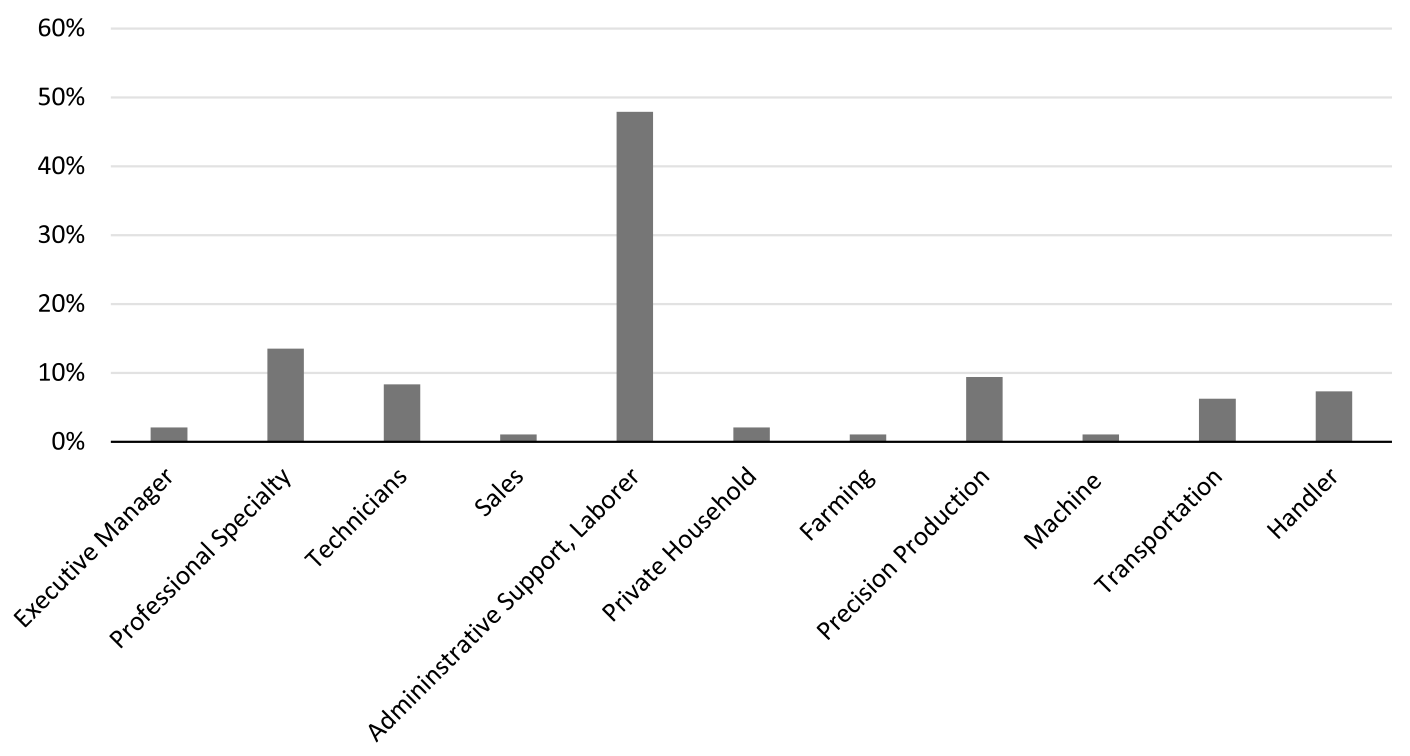

Fig. 2. Occupational categories for successfully closed cases.

one point increase in MPAI-4 T score (higher scores indicating a greater level of disability), the odds of successful employment declines by nearly one percent (Wald =9.06, $p=.003$ ) (See Fig. 4).

Adding treatment group to the model and leaving level of disability as a covariate increased the models significance and improved the odds of successful employment for those in the treatment group. This model was also statistically significant, $\chi^{2}(2)=14.41, p=0.001$ and explained $8 \%$ of the variance in productive activity and correctly classified

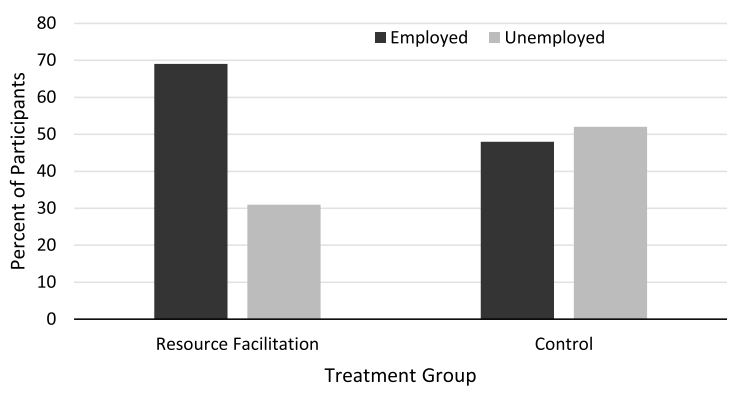

Fig. 3. Employment Rates for RF and Control Groups. 


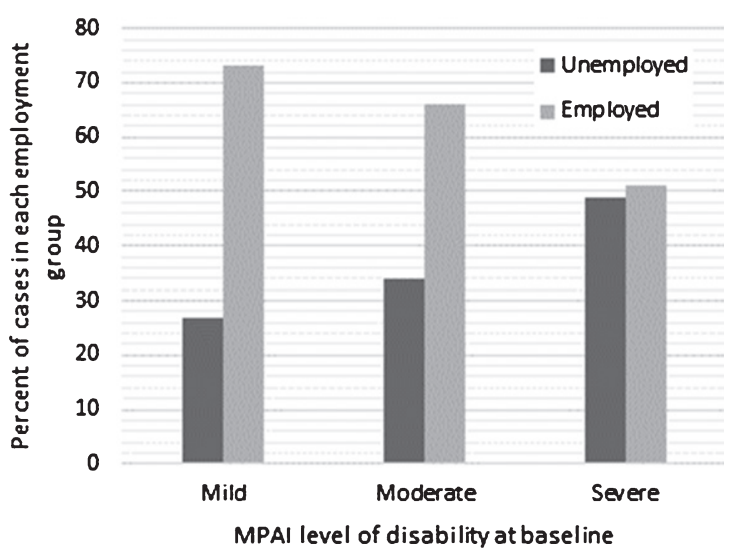

Fig. 4. Outcome by Initial Level of Disability.

Table 3

Summary of logistic regression analysis predicting employment

\begin{tabular}{lcccc}
\hline Variable & $B$ & SE & Wald Statistic & $p$ \\
\hline Treatment Group & -0.824 & 0.388 & 4.52 & 0.033 \\
Disability & -0.056 & 0.019 & 8.51 & 0.004 \\
\hline
\end{tabular}

$67.1 \%$ of the cases. When controlling for baseline level of disability, treatment group significantly predicted employment outcome (Wald $=4.52, p=0.033$ ) and participants in the treatment group were 2.3 times more likely to return to work than controls.

\section{Conclusions}

In conclusion, participants receiving RF had a higher employment rate than participants in past control groups. In fact, participants in the treatment group were over two times more likely to return to work than the control group when controlling for level of disability. As demonstrated by previous research, successful vocational outcome varied as a function of level of disability as measured by the MPAI-4, where $74 \%$ of participants with mild disability, $65 \%$ of participants with moderate disability, and $48 \%$ of participants with severe disability were successful with obtaining employment through RF. Further, the participants in RF were almost ten years postinjury as compared to the control group, which was slightly over two months post-injury. Additionally, the RTW rate of $69 \%$ for the RF cohort is considerably better than what the literature would suggest for people of ABI of approximately $40 \%$ for all levels of disability. The findings from the present study are also consistent with the RTW rates we found in our two previous RCT's. The present findings provide some initial support for the clinical effectiveness of RF for a sample referred by the Indiana Vocational Rehabilitation Services.

However, the present study has several limitations. Specifically, the sampling method in this study is not traditional, and although our control group was randomized into the control condition, our treatment group was not, therefore potentially adding bias to our study. In addition, some significant differences between the groups at baseline could confound some of the outcome variables, specifically time since injury, age at injury, as well as diagnosis. However, this sample is more representative of a clinical population seen in a State Vocational Rehabilitation agency, and likely better represents an otherwise ignored sector of the population previously underrepresented in clinical trials. Therefore, these findings support the transition from the established RF efficacy into clinical effectiveness.

The present study took place over different economic epochs, which could serve to influence RTW. The recession occurred from 2007 through 2011. Control group data was collected between 2008 and 2013, while data from the treatment group was collected between 2009 and 2012. Therefore, a larger proportion of data collection took place during the recession for the treatment group than the control groups. Therefore, it would seem unlikely that the changes in the economy served as a positive bias for RTW for the treatment group.

Although this study successfully demonstrates the effectiveness of RF, additional established predictors of employment success should be taken into account. For example, Cuthbert and colleagues found that age, race, gender, marital status, rehabilitation payment source, education, pre-injury work status, length of stay, and the disability rating scale (DRS) were significant predictors of employment two years post injury (Cuthbert et al., 2015). Due to our sampling methods, we were unable to use well-known predictors of outcome in our statistical analyses. This exclusion of key predictors likely led to the small effect size of our prediction model. Also, future studies in RF need to consider relationship between pre-injury rates of employment and post-RF employment and study change in occupational type from pre-injury to postinjury. Last, we were not able to evaluate durability or stability of employment for more than 90 days, and given that the disability associated with these injuries was likely chronic, and for some, the level of disability might get worse. This would therefore 
suggest a need for long-term surveillance to proactively monitor vocational adjustment and stability.

\section{Acknowledgments}

The authors would like to acknowledge Peter A. Bisbecos, J.D., former Director of the Division of Disability \& Rehabilitative Services, and Kylee B. Hope, J.D., current Director of the Division of Disability \& Rehabilitative Services, whose support provided the opportunity to complete the present prospective clinical cohort study. The authors would also like to acknowledge the efforts of Summer Ibarra, Ph.D., Laura C. Trexler, O.T.R., Judy Reuter, and the entire Resource Facilitation team who provided the services to the participations in the present study.

\section{Conflict of interest}

The authors declare that they have no conflicts of interest.

\section{References}

American Medical Rehabilitation Providers Association, eRehab-data. (2012). Retrieved from https://web2.erehabdata. com/erehabdata/index.jsp

Ben-Yishay, Y., Silver, S. M., Piasetsky, E., \& Rattok, J. (1987). Relationship between employability and vocational outcome after intensive holistic cognitive rehabilitation. The Journal of Head Trauma Rehabilitation, 2(1), 35-48.

Bounds, T. A., Schopp, L., Johnstone, B., Unger, C., \& Goldman, H. (2003). Gender differences in a sample of vocational rehabilitation clients with TBI. NeuroRehabilitation, 18(3), 189-196.

Brain Injury Association Inc. (2001). Resource Facilitation: A consensus of principles and best practices to guide program development and operation in brain injury. Alexandra, VA: Connors, S.H.

Corrigan, J. D., \& Hammond, F. M. (2013). Traumatic brain injury as a chronic health condition. Archives of Physical Medicine and Rehabilitation, 94(6), 1199-1201.

Cuthbert, J. P., Harrison-Felix, C., Corrigan, J. D., Bell, J. M., Haarbauer-Krupa, J. K., \& Miller, A. C. (2015). Unemployment in the United States after traumatic brain injury for working-age individuals: Prevalence and associated factors 2 years postinjury. The Journal of Head Trauma Rehabilitation, 30(3), 160-174.

Dikmen, S., Machamer, J., \& Temkin, N. (1993). Psychosocial outcome in patients with moderate to severe head injury: 2-year follow-up. Brain Injury, 7(2), 113-124.

Dikmen, S. S., Temkin, N. R., Machamer, J. E., Holubkov, A. L., Fraser, R. T., \& Winn, H. R. (1994). Employment following traumatic head injuries. Archives of Neurology, 51(2), 177-186.
Fadyl, J. K., \& McPherson, K. M. (2009). Approaches to vocational rehabilitation after traumatic brain injury: A review of the evidence. The Journal of Head Trauma Rehabilitation, 24(3), 195-212.

Groswasser, Z., Reider-Groswasser, I. I., Schwab, K., Ommaya, A. K., Pridgen, A., Brown, H. R., . . \& \& Salazar, A. M. (2002). Quantitative imaging in late TBI. Part II: Cognition and work after closed and penetrating head injury: A report of the Vietnam Head Injury Study. Brain Injury, 16(8), 681-690.

Kolakowsky-Hayner, S. A., \& Kishore, R. (1999). Caregiver functioning after traumatic injury. NeuroRehabilitation, 13(1), 27-33.

Kreutzer, J. S., Marwitz, J. H., \& Kepler, K. (1992). Traumatic brain injury: Family response and outcome. Archives of Physical Medicine and Rehabilitation, 73(8), 771-778.

Kreutzer, J. S., Gervasio, A. H., \& Camplair, P. S. (1994). Primary caregivers' psychological status and family functioning after traumatic brain injury. Brain Injury, 8(3), 197-210.

Malec, J. F., Buffington, A. L., Moessner, A. M., \& Degiorgio, L. (2000). A medical/vocational case coordination system for persons with brain injury: An evaluation of employment outcomes. Archives of Physical Medicine and Rehabilitation, 81(8), 1007-1015.

Malec, J. (2005). The Mayo Portland Adaptability Inventory. The Center for Outcome Measurement in Brain Injury. Retrieved July 27, 2017 from http://www.tbims.org/combi/mpai

Malec, J. F., \& Moessner, A. M. (2006). Replicated positive results for the VCC model of vocational intervention after ABI within the social model of disability. Brain Injury, 20(3), 227-236.

Prigatano, G. P., Fordyce, D. J., Zeiner, H. K., Roueche, J. R., Pepping, M., \& Wood, B. C. (1984). Neuropsychological rehabilitation after closed head injury in young adults. Journal of Neurology, Neurosurgery \& Psychiatry, 47(5), 505-513.

Trexler, L. E., Trexler, L. C., Malec, J. F., Klyce, D., \& Parrott, D. (2010). Prospective randomized controlled trial of resource facilitation on community participation and vocational outcome following brain injury. The Journal of Head Trauma Rehabilitation, 25(6), 440-446.

Trexler, L. E., Parrott, D. R., \& Malec, J. F. (2016). Replication of a prospective randomized controlled trial of resource facilitation to improve return to work and school after brain injury. Archives of Physical Medicine and Rehabilitation, 97(2), 204-210.

UDS. (2012). Uniform Data System for Medical Rehabilitation. Retrieved from http://www.udsmr.org/Default.aspx

Van Velzen, J. M., Van Bennekom, C. A. M., Edelaar, M. J. A., Sluiter, J. K., \& Frings-Dresen, M. H. W. (2009). How many people return to work after acquired brain injury?: A systematic review. Brain Injury, 23(6), 473-488.

Wehman, P., Bricout, J., Targett, P., Fraser, R. T., \& Clemmons, D. C. (2000). Supported employment for persons with traumatic brain injury: A guide for implementation. Traumatic Brain Injury Rehabilitation, 201-240.

Wehman, P., West, M., Fry, R., Sherron, P., Groah, C., Kreutzer, J., \& Sale, P. (1989). Effect of supported employment on the vocational outcomes of persons with traumatic brain injury. Journal of Applied Behavior Analysis, 22(4), 395-405.

Wehman, P., Kregel, J., Keyser-Marcus, L., Sherron-Targett, P., Campbell, L., West, M., \& Cifu, D. X. (2003). Supported employment for persons with traumatic brain injury: A preliminary investigation of long-term follow-up costs and program efficiency. Archives of Physical Medicine and Rehabilitation, 84(2), 192-196. 